\title{
Post-flare Coronal Activity on AU Mic Detected by EUVE
}

\author{
Maria Katsova ${ }^{1}$, Jeremy Drake ${ }^{2}$, Moissei Livshits ${ }^{3}$ \\ 1 Sternberg State Astronomical Institute, Moscow State University, 119899 Moscow, \\ Russia \\ 2 Center for EUVE Astrophysics, University of California, Berkeley, CA 94720, USA \\ 3 Institute of Terrestrial Magnetism, Ionosphere, and Radio Wave Propagation, \\ Russian Academy of Sciences, 142092 Troitsk, Moscow Region, Russia
}

Data of long-duration emission arising after the impulsive rise and decay in a flaring event on the red dwarf star AU Mic are discussed. Intensive EUV emission in the band $65-190 \AA$ was registered by the Extreme Ultraviolet Explorer $(E U V E)$ after both impulses during half a day. A similar behavior of the flux in the FeXVIII $93.9 \AA$ line is detected after the first powerful impulse. The decay of the intensity in the 65-190 $\AA$ band and in the Fe XVIII line during this prolonged event is $\mathbf{1 0}$ times slower than the time of radiative cooling of coronal loops with a typical flare plasma density. Some difficulties with two explanations of this event proposed earlier are discussed. Explanation (i) - the radiation of dense loops at main phase of the flare, and (ii) - the emission of the low-dense plasma of coronal transients (CME). The temporal behavior of the emission measure is determined for both the 65-190 $\AA$ band and the Fe XVIII line fluxes. The total energy emitted in the 1-2000 $\AA$ region for the long-duration event lasting almost 12 hours is $3 \cdot 10^{35}$ ergs. The following physical model is proposed to explain the prolonged event (Fig. 1): the source of emission is the system of high coronal loops, the size of which is more than the active region scale, but is less than the stellar radius. The temperature of the plasma in the loops decreases from $10^{7} \mathrm{~K}$ slowly, during a few hours. The densities in these loops are in the range $10^{13} \mathrm{~cm}^{-3}$ to $5 \cdot 10^{9} \mathrm{~cm}^{-3}$. Such systems, when the plasma therein becomes cool, are observed in the $\mathrm{H} \alpha$ line during large solar flares (for instance, June 15, 1991) after CME. Some additional post-flare energy input into this high coronal loop systems can be caused by the reconnection in vertical current sheet, and this post-eruptive energy release provides prolonged and intensive EUV emission.

Apparently, we are faced here with a new kind of surface activity on latetype stars which is intermediate between impulsive flares on red dwarfs and long-duration, powerful events on subgiants, which are components of RS CVn binaries.

The full version of this contribution will be published in Astronomicheskij Zhurnal (Astronomy Reports), 1995, Vol. 72. 


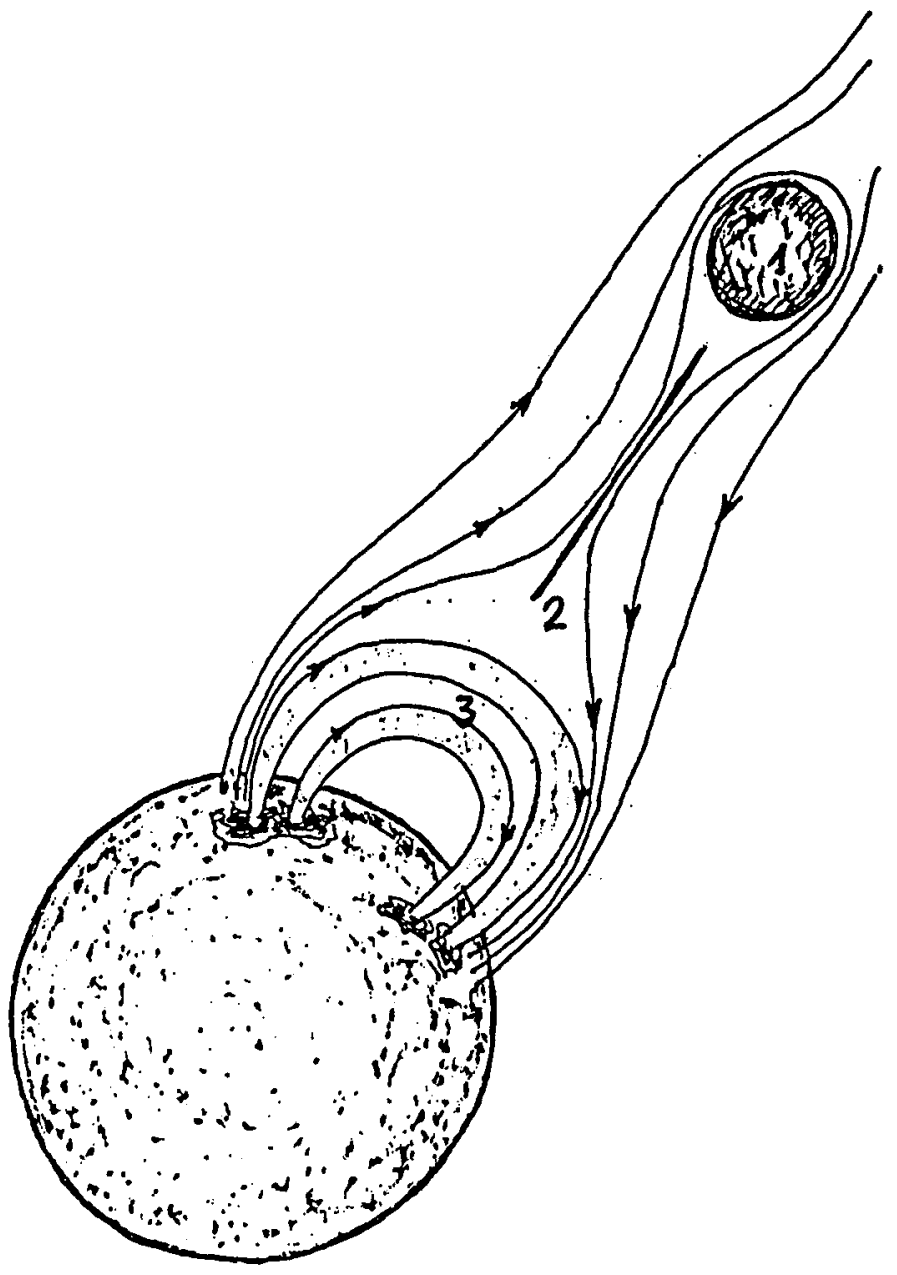

Fig. 1. The event with post-eruptive energy release: (1) - coronal mass ejection (CME), (2) - vertical current sheet, (3) - coronal loops as a source of the EUV radiation 